\title{
ADDITIONAL OBSERVATIONS
}

\author{
on
}

\section{OBSTRUCTIONS OF THE PULMONARY ARTERIES.}

BY JAMES PAGET, F.R.C.S., LECTURER ON PHYSIOLOGY, AND WARDEN OF THE COLLEGE AT ST. BARTHOLOMEW'S HOSPITAL.

Received May 22nd-Read June 24th, 1845.

THE following case of obstruction of the branches of the pulmonary artery by clots of blood, will, I hope, be deemed of interest sufficient to justify its admission, together with some remarks suggested by it, into the Transactions of the Society, as an appendix to the paper on the same subject, in the last volume. It presents the disease in a new and important form, making its progress unattended by alarming signs, and almost unobserved, and then destroying life with terrible abruptness.

A ruined wine-merchant, 55 years old, and who had drunk hard, had been in St. Bartholomew's Hospital, under the care of Mr. Stanley, since the 18th of April, suffering with stricture of the uretbra and paralysis of the bladder. He was a bloated, unhealthy-looking man, and had occasionally slight cedema of the hands and feet; but he was so nearly well, that he never complained to any of his attend-

voL. XXVIII.

2 A 
ants of more than the disorder of his urinary organs. Once, the house-surgeon, Mr. Parson, noticed that he had a cough; and he said that he was often subject to such an one, but thought it trivial. After his death, too, I learned from his fellowpatients that, in the last week of his life, he had sometimes spoken of having occasional shortness of breath, so that when he wished to sleep in the day, he was "apt to be taken with a panting;" and such an attack came on once or twice while he was walking in the ward. But these attacks were rare; they lasted only a short time, and they never appeared so serious as to elicit more than a passing remark from him; he mentioned them to none but the other patients in the ward; and within two hours of his death he talked in the same good spirits, in which he had been all day, of what he would do when he left the hospital with his stricture cured. On the morning of the 4th of May, the sister of the ward thought him looking unusually well. In the afternoon, after a short sleep, he proposed to a patient near him to have tea; but first he went to the water-closet, and, on his return, presenting nothing unusual in his appearance, he sat down on his bed-side. A minute after, he fell heavily on his face; and, with a slight distortion of his mouth and of one arm, died before two minutes more bad elapsed.

The body was examined twenty-two hours after death.

The skull and brain presented nothing unna- 
tural, except a few small earthy masses in the choroid plexuses. The cerebral substance had an ordinary appearance of vascularity.

The larynx, trachea and bronchi appeared healthy, their mucous membrane was pale. Some of the large bronchi contained a frothy mucus, slightly tinged with blood. The left pleural cavity was obliterated by general and rather close adhesions, the right was free, and contained a small quantity of fluid. The lungs were large and deep-coloured, but externally, appeared nearly healthy: their upper lobes were œdematous; the middle and lower lobes partially emphysematous, and containing a moderate amount of blood and serum. There was in no part of either lung a trace of pulmonary apoplexy, or of intense congestion. In the apex of the right lung was a slightly depressed cicatrix over a few dry tubercles, and a small healing cavity, full of thick tuberculous, and soft mortar-like substance.

The cavities of the right auricle and ventricle were large: their walls were of little, if at all, more than the natural thickness, but of firm and healthy texture. They contained a moderate quantity of fluid blood, with soft clots, and a mass of firm, colourless, semi-transparent coagulum, like a buffy coat, a prolongation from which, extended from the right auricle into the vena cava superior and some of its branches. The tricuspid and pulmonary valves were healthy, and so was the pulmonary artery, in the size and texture of both its trunk and all its visible branches. But nearly all the branches 2 A 2 
beyond the primary divisions of the pulmonary artery contained clots of blood, which, from a comparison of them with those found in tied arteries, I judged to be from three to ten days old, those of the least age being in the largest branches. They were firm, cylindroid, and of various colours, black, brownish, pink, and yellow ochre, irregularly mixed. Some of them were much drier than recent clots, and grumous; others appeared invested and intersected by thin layers of white fibrine. Most of them filled the portion of the artery in which they lay, some did so to distension; a few of the driest and most decolorized adhered to the walls of the vessel, and left it rough and blood-stained when they were removed. The clots did not commonly extend continuously from any large branch of the pulmonary artery into many of its successively subordinate divisions; some of them were in the shape of thick cylinders, from half an inch to an inch in length, which filled a branch of the artery, but were not even in contact with the similar clots which filled adjacent branches; some extended from a larger branch into two or three smaller ones derived from it, filling them all, but ending abruptly; only a few branched many times, and filled a larger series of the branches of the artery. No branch of the pulmonary artery less than half a line in diameter appeared to contain any of these clots, and the pulmonary veins were healthy and empty.

The left auricle and ventricle were larger, and their walls were thicker, than in the healthy state. 
They contained a small quantity of black fluid blood. The mitral valves were opaque, but pliant, and probably efficient. The aortic valves were in a similar condition, and of large size, adapted to the aorta itself, which was uniformly slightly dilated, and thickly beset with fatty, and a few earthy deposits in its coats. The large systemic arteries and veins were all healthy and empty, or containing a little fluid blood.

The whole digestive canal was healthy. Digestion appeared to have been active at the time of death, in both the stomach and the small intestines. The liver and pancreas were healthy : the spleen was of middle size, and soft.

In both kidneys, the pelvis and infundibula were dilated, the papillæ flattened and expanded, and the texture generally, but not uniformly, indurated and contracted, so that their external surface was lobed, and in parts coarsely granular. The ureters were dilated and tortuous, the bladder very large, and its walls thin and weak.

Behind the pharynx, near the right sterno-clavilar articulation, and in the course of the right femoral artery, there were small effusions of blood, like bruises, in the loose fibro-cellular tissue; but with these exceptions, no part of the body appeared as if peculiar or general congestion had existed.

Among the points suggested for consideration by this case, there are two of peculiar physiological interest, on each of which I beg to offer some remarks, 
namely, the mode of death, and the probable cause of the coagulation of blood during life.

The case proves that, in certain circumstances, a great part of the pulmonary circulation may be arrested in the course of a week, (or a few days more or less,) without immediate danger to life, or any striking indication of what has happened. And the evidence of this case is confirmed by others of similar obstruction of the pulmonary arteries. This is, indeed, the only recorded instance of the disease being suddenly fatal in a person, who just before his death appeared in tolerable health ; yet in some others it is certain that many of the pulmonary arteries must have been impervious long before the patient, though seriously ill, presented any distinct signs of such a hindrance to the circulation. For examples, among those whose cases are mentioned in my former paper, one* was suddenly seized with the signs of the obstruction during convalescence after a severe operation, and died in a few hours; in another, $\uparrow$ the fatal attack came on and terminated as speedily, during convalescence after pneumonia; a third patient $\ddagger$ had long been greatly exhausted, but had no dyspnœa till shortly before her death; a

* Vol. xxvii., p. 167.

† Case from M. Baron, ibid., p. 164. [Since this was written, a somewhat similar case has been published by $M$. Richelot in the Gazette des Hopitaux, 29 Avril, 1845. The patient, a man 38 years old, appeared convalescent from pleuro-pneumonia, when suddenly he was attacked with a feeling of suffocation, and soon died.]

† Ibid., p. 182. 
fourth* had various signs of dangerous import, resulting chiefly from obliterations of large systemic veins, but no dyspnœa till within an hour and a half of her death : yet in all these cases the characters of the clots by which the pulmonary arteries were obstructed, showed plainly that they had been for a week or more in progress of formation. Moreover, it may be observed that many if not all of those patients in whom the respiration was much affected during the formation and subsequent changes of the clots, had other extensive disease of the heart or lungs, to which the greater part of the dyspnœa might be ascribed. $\dagger$ It appears, therefore, that the obstruction of a large and quickly increasing portion of the pulmonary circulation, if it be not complicated by other disease, is usually unattended by disturbance of the respiration or any other important function.

Strange as this fact may at first seem, it may be explained : and its explanation will guide to that of the manner in which the increasing obstruction of the pulmonary arteries at last destroys life.

The absence of any constant deep distress of breathing during an extensive closure of the pulmonary arteries, shows that there can be no constant or

* M. Lediberder's case related by M. Baron in his " Recherches sur la coagulation du Sang dans l'Artére Pulmonaire," in the Archives Générales de Médecine, Paris, 1838, t. iii., p. 18.

+ See the cases related in the former paper at pages 168,171 , 178 , and a case by Dr. C. J. B. Williams, reported in the Medical Gazette, November 22, 1844, p. 257. 
great congestion of those vessels of the lungs which are still able to convey blood ; and the healthy manner in which, in some of the cases, the functions of other organs were discharged, makes it probable that all the systemic vessels were equally free from congestion. Now, for the avoidance of either general or pulmonary congestion, it is essential that equal quantities of blood shall in a given time pass through the systemic and pulmonary circulations respectively. To this end the conditions of the healthy state are exactly adapted ; and in nearly all diseases of the respiratory and circulatory systems, a natural tendency to the preservation of the balance may be observed.

In the case of an obstruction of the pulmonary arteries-provided it is of small extent-the right ventricle will naturally increase the velocity of the blood, which it propels through the remaining open arteries; and it may so do this, and, at the same time, so dilate these vessels, that the quantity passing through the lungs in a given time shall not be diminished; in which case no change will be needed in the systemic circulation. But when many and considerable branches of the pulmonary artery are obliterated, and the usual quantity of blood cannot, in the usual time, by the aid of the compensations just mentioned, be transmitted through the lungs, then, the balance of the two circulations can be maintained, and congestion can be avoided, only by the movement of the blood through the systemic vessels being retarded, till the quantity traversing them in a given time is not greater than the reduced quantity which 
now, in the same time, traverses the lungs. ${ }^{*}$ This adjustment will be naturally effected-for, when the left ventricle receives, through those pulmonary arteries which remain open, a smaller than the usual quantity of blood at each dilatation, it must, in each contraction, discharge an equally small quantity; and the consequence of this (if other circumstances remain the same) must be a diminished velocity of the movement of the blood through the systemic vessels.

The peculiarity, therefore, of a person who has a considerable portion of his pulmonary arteries obstructed, must be, that, in direct proportion to the extent of the obstruction, his systemic circulation is less rapid, and his remaining pulmonary circulation more rapid, than before the obstruction took place. In such a state, it might be expected (and the cases prove) that the patient would live for a time without serious discomfort; for other facts show that an unusual rate of movement of the blood through a part, if unattended by distension of the vessels, does not immediately affect its function. Besides, although only a small quantity of oxygen may enter the blood, yet during rest a small quantity is sufficient for health; and there is no unusual retention of carbonic acid, for all the blood which enters the

* At least, this is the only way in which the balance of the circulations can be preserved, if, as in the recorded cases, neither the whole quantity of circulating blood, nor the quantity, whether moving or at rest, in the lungs, is materially reduced. 
left side of the heart will, in the absence of any disease of the lungs themselves, have been sufficiently exposed to the atmosphere.

But such a state, though not necessarily one of discomfort, must always be one of danger; for if, after the circulation through those pulmonary arteries which remain open, has attained its maximum velocity, the circumstances which first led to the coagulation of the blood continue, then, as the obstruction by clots forming in the pulmonary vessels increases, so must the retardation of the blood in the systemic vessels. And this will go on till the blood ceases, or so nearly ceases, to flow in the systemic vessels, that the actions of the heart and nervous centres are no longer maintained; and thus the patient suddenly or slowly dies.

It thus appears probable, that the direct cause of death in these cases is not, as one might expect, in the state of the lungs, but in that of the brain or of the heart : the patient dies because these organs are not supplied with fresh blood. The mode of death is altogether peculiar. It is, in some respects, like death by asphyxia : in both, there is an arrest of blood at the lungs, but in this disease, the blood which does pass through them is aerated; in asphyxia it is not, and in it there are, therefore, all the signs of poisoning by carbonic acid, and the agony of want of breathing. Again, this mode of death resembles, in some points, death by anæmia; in both, the organs die for want of fresh supplies of 
blood, but in anæmia there is no blood for them; in these cases, there is blood even in the organs, but it is not constantly renewed.

It accords with this explanation of the mode of death, that it is sometimes gradual, the movement of the systemic blood being gradually arrested; and that, sometimes, when the obstruction is very extensive, death ensues suddenly from an accidental event, such as unusual muscular exertion, which, by accelerating the movement of the venous blood, may discharge the systemic vessels more rapidly than they can be filled again through the remaining open pulmonary vessels.* Thus, the subject of this paper died directly after the exertion of defecation and walking; another was suddenly seized while walking, during convalescence.

In many cases, this mode of death is complicated, and accelerated by the effects of other diseases. When, together with the obstruction of the pulmonary arteries, patients have extensive bronchitis, or œdema, or other structural diseases of the lungs, as-

* Of the patients whose cases have been hitherto recorded, fourteen in number, six have died suddenly, namely, the subjects of M. Baron's 2nd, 3rd, and 4th cases; those of the 2nd case in my former paper (p. 167), and of this case; and a patient whose history is briefly given by Dr. Bright (Medical Reports, vol. i. p. 19); and to these may now be added M. Richelot's case,-making seven sudden deaths in fifteen cases. In some of the others, sudden attacks, or great aggravations of dyspncea, or sinking, such as in a rather worse degree might have proved suddenly fatal, have been observed: see especially the 5 th case in my former paper, p. 174. 
phyxia may add its signs, and quite overwhelm those of the ceasing movement of the blood. Several of the cases show this very plainly; and others show, that when the lungs are in their own texture healthy, death, whether gradual or sudden, is tranquil.

With respect to the second point proposed for consideration-the cause of the coagulation of the blood in the pulmonary arteries-if all the recorded cases of this disease be brought together, there will be found great diversity in the circumstances leading to the formation of the clots.

In some cases, it appears almost certain that the chief reason why the blood coagulates is, that its passage through the small vessels is arrested by disease of the left side of the heart, or by pneumonia, pulmonary apoplexy, or some other disease external to themselves obstructing them,* or by some morbid substance collecting in them. $\dagger$

In other cases, the coagulation may be chiefly ascribed to the blood almost ceasing to be moved by the weakened hearts of those who, by long-continued disease of any kind, have become utterly exhausted ; a condition which, like the preceding, will also greatly increase the chances of coagulation taking place, when it coincides with any other of the circumstances in which it may happen.

* Such are M. Baron's 2nd case, p. 17 ; those referred to in the former paper, pp. 163, 168, 171, 173; Dr. Williams's case.

$+\mathrm{As}$ in the three cases in the former paper, pp. 165, 166.

$\ddagger$ Such, perhaps, as the patient in the 6th case, in the former paper, p. 182, and M. Baron's 3rd case, 1. c., p. 9. 
Again, in other cases the blood coagulates in inflamed pulmonary arteries, as it does in inflamed veins ;* and, probably, it would coagulate in any pulmonary artery whose coats were much roughened by disease. $\dagger$

But in the case just recorded, and in at least four others, $\ddagger$ the blood appears to have coagulated, independent of all these conditions; the texture of the lungs was, if at all, only trivially diseased; the coats of the obstructed pulmonary arteries were healthy, the heart has appeared sufficiently powerful, and the patients have shown no signs of extreme exhaustion before, or while the clots were forming. In these mysterious, but most interesting cases, therefore, we must look for explanation to the condition of the blood itself.

It is very important to observe that in all these cases, though there was no manifest mechanical hindrance to the movement of the blood, the clots were in masses, not laminated ; indicating that the blood, when it coagulated, must have been at rest. And that it was at rest, both during and after coagulation, is further evidenced by the older clots in this

* Cruveilhier, Anatomie Pathol., Livr. xi., Pl. 2, 3 ; and otbers.

$\dagger$ In M. Baron's 4th case, 1. c., p. 22, this may have been the cause of the coagulation.

$\ddagger$ M. Baron's lst case, l. c., p. 6; Dr. Bright's case, 1. c.; and the 4 th and 5 th cases in my former paper, pp. 174, 178. (Probably, also, M. Richelot's case, already cited, may be added to these, for the texture of the lungs is not said to have been materially changed.) 
and a former case being. in numerous pieces, not branched and extending continuously through all the branches of the arteries in which they were found; for this must be ascribed to the breaking of a large continuous clot, (such as could only be formed during rest,) in the act of contraction which succeeded to the simultaneous coagulation of the whole mass. Now, rest being a sufficient condition for the coagulation of ordinary blood, we need not suppose that, in these cases, there was such a morbid state of the blood as rendered it peculiarly coagulable; especially when such a supposition is contradicted by its fluid, or softly coagulated state, in all the vessels except the pulmonary arteries. Neither can it be supposed that the blood ceased to be moved or coagulated because portions of it died ; for, besides that it is probable that coagulation itself is partial organization, there is evidence that, in one of the cases, ${ }^{*}$ the blood after coagulation became more highly organized.

In the absence, then, of all evidence of a peculiar disposition of the blood to coagulate, and in the assurance that the coagulation took place during the rest of the blood, although no cause of obstruction external to the vessels could be discerned, I will suggest, that the chief cause of the stoppage of the blood, and of its consequent coagulation, in these cases, is some morbid state affecting its constitution, so as to increase that adhesion of it to

* Case V., and Pl. III. fig. 3, in the former paper. 
the walls of the vessels which constitutes, even in the healthy state, the greatest resistance which the heart's power has to overcome.

In this view, the frequent coincidence of granular degeneration of the kidneys with old clots in the pulmonary arteries is interesting.* Many of the signs of that disease of the kidneys, and especially the enlarged heart, and the dilated and tortuous arteries so often found, indicate that there is an unnatural difficulty in the transmission of the blood through the capillaries or the small arteries and veins. And to explain this difficulty, it is not unreasonable to suppose that the diseased blood adheres more than healthy blood does to the walls of the vessels, the urea or some other retained constituent of the urine acting in the same manner as experiments $\dagger$ have shown that salts of baryta and some other bases will act, which, when introduced in even very small quantities into the blood, hinder, or altogether prevent its passage through the small vessels, although the heart be acting with full force.

On this hypothesis, all the circumstances of these cases may be explained. At first, we may suppose

* The coincidence existed in at least three of these five cases, viz. : in this present case, in Dr. Bright's, and in Case V.p. 178, in the former paper : in the other two cases, the state of the kidneys is not mentioned. They were granular in Cases II. and III. in that paper, and may have contributed to the coagulation of the blood, although the state of the heart and lungs may have been an equally or more favourable condition for that result.

† Especially those of Mr. James Blake. 
all the blood moving at an equally retarded rate through the pulmonary capillaries. At length some of it ceasing to move in a part where an accidental congestion, however slight, makes its passage more peculiarly difficult, clots will be formed in the corresponding pulmonary arteries, to which result also, the absence of anastomoses between the larger branches will contribute. After the obstruction of a part of the pulmonary circulation, a larger portion of the force of the right ventricle will be available for the rest : this, therefore, may go on at least as well as before, till, the evil condition of the blood continuing, it ceases to move in some other part, and fresh clots form. Thus the disease may make progress till, in either of the ways already spoken of, death ensues, or, as has happened in at least one case, ${ }^{*}$ the blood may regain a healthier state, and the obstruction and coagulation ceasing, the clots already formed, acquire higher organisation, and, adhering to only one part of the arteries which they filled, may allow blood again to flow through them : thus the patient may recover.

In conclusion, I will venture to suggest that many cases of sudden death, for which no cause has been found, or which have been ascribed to some insufficient or improbable cause, have depended on clots obstructing the pulmonary arteries; and that the clots of the same kind, which are often found in the systemic veins, and are usually ascribed to

* Case V. p. 178, in the former paper. 
phlebitis, though the coats of the veins are healthy, are the consequence of stoppage of the blood similar to this which I have described, and from a similar cause. It is no argument against the supposition of the frequency of such arrests or slow movements of the blood that the pulse continues : for the pulse is not, necessarily, an indication that the blood is flowing through the vessels, any more than a wave is sure evidence of a tide.

P.S. Since the preceding paper was read to the Society, I have met with the observations of my esteemed friend Dr. Angelo Dubini on the same subject. These are contained in an essay full of good practical pathology, which was read to the medical section of the Scientific Congress at Milan, in September 1844, and are published with an appendix containing a translation of the abstract of my former paper, in the "Annali Universali di Medicina, di Febbrajo, 1845."

Dr. Dubini states that he has been in the habit of examining clots in the branches of the pulmonary arteries since 1839, when his attention was first called to them by a memoir which was read by Dr. Alessandro Gambarini at a monthly meeting at the Milan Hospital.* He says that my observations are in almost perfect agreement with his own and Dr. Gambarini's; but I regret that he has given so few particulars of his cases, that the

* Dr. Dubini does not refer to this memoir as one published, neither can I find it in any of the Journals of 1839 or 1840.

vol. XXVIII. 2 B 
following are the only matters of fact directly bearing on the subject, which I can gather from his own essay, and his commentaries on mine.

$\mathrm{He}$ has found in "surprising frequency," clots obstructing the branches of the pulmonary arteries, and presenting all the characters of clots formed in vessels at various periods before death. In a series of twelve cases, the lungs in eight presented no such change in their proper texture as could give rise to obstruction of the capillary circulation. "In some of them there was slight pulmonary œdema : in others some engorgement by bloody serum; in others the texture of the lungs was soft and dry; in one there were clots in the arteries of one lung which was not œdematous, while the other, which was œdematous, contained none. Of the four cases in which the pulmonary texture was in some degree affected, two had circumscribed or lobular hepatization with gangrenous excavations, the two others had phthisis with tuberculous cavities. In most cases the clots occupied the large divisions of the pulmonary artery, while the trunk and secondary branches were free."

Pulmonary emphysema, organic disease of the heart, spots of yellow deposit in the inner coat of the pulmonary arteries, and dilatation of the branches thus diseased, were frequent concomitants of the obstructions; but no similar clots were found in cases of hepatization.

In endeavouring to explain the formation of clots in the pulmonary arteries, Dr. Dubini rejects the supposition of an inflammation (like phlebitis) of 
the obstructed vessels, as an improbability. He suggests that the cause of the coagulation during life is in the altered crasis of the blood; but he thinks that there are many facts in opposition to the theory, "which destroys every hope of success in the explanation of the genesis of the clots." 